\title{
A Simplified Spin-Flip Time-Dependent Density Functional Theory (SF-sTD-DFT) Approach for the Electronic Excitation Spectra of Very Large Diradicales Supplemental Information
}

\author{
Marc de Wergifosse ${ }^{\dagger *}$, Christoph Bannwarth ${ }^{\$}$, Stefan Grimme $^{\dagger}$ \\ ${ }^{\dagger}$ Mulliken Center for Theoretical Chemistry, Institut für Physikalische \\ und Theoretische Chemie, Beringstr. 4, 53115 Bonn, Germany \\ ${ }^{\$}$ Department of Chemistry, Stanford University Stanford, CA 94025, USA
}




\section{Appendix}

To understand why we included a factor $\sqrt{2}$ to equation 10 from the manuscript, one can rewrite this expression without this factor in terms of 1-electron transition density matrix (1-TDM), considering that $m \neq n$ :

$$
\left\langle m\left|\mu_{\xi}\right| n\right\rangle=\frac{1}{2}\left\{\sum_{i_{\alpha} j_{\alpha}}\left[\mu_{i_{\alpha} j_{\alpha}, \xi} \gamma_{i_{\alpha} j_{\alpha}, o c c .}^{m n}\right]+\sum_{a_{\beta} b_{\beta}}\left[\mu_{a_{\beta} b_{\beta}, \xi} \gamma_{a_{\beta} b_{\beta}, \text { unocc. }}^{m n}\right]\right\}
$$

where the spin-flip state to spin-flip state 1-TDM is composed of two occupied-occupied (occ.) and unoccupied-unoccupied (unocc.) blocks:

$$
\gamma_{S F}^{m n}=\left(\begin{array}{cc}
\gamma_{o c c .}^{m n} & 0 \\
0 & \gamma_{u n o c c .}^{m n}
\end{array}\right)
$$

where

$$
\gamma_{i_{\alpha} j_{\alpha}, o c c .}^{m n}=\left\langle m\left|j_{\alpha}^{+} i_{\alpha}\right| n\right\rangle=\sum_{a_{\beta}}\left[X_{i_{\alpha} a_{\beta}}^{n} X_{j_{\alpha} a_{\beta}}^{m}\right]
$$

and

$$
\gamma_{a_{\beta} b_{\beta}, \text { unocc. }}^{m n}=\left\langle m\left|b_{\beta}^{+} a_{\beta}\right| n\right\rangle=-\sum_{i_{\alpha}}\left[X_{i_{\alpha} a_{\beta}}^{n} X_{b_{\beta} i_{\alpha}}^{m}\right] .
$$

To evaluate the oscillator strength, one needs to evaluate:

$$
\left\langle n\left|\mu_{\xi}\right| m\right\rangle\left\langle m\left|\mu_{\xi}\right| n\right\rangle=\frac{1}{4}\left\{\begin{array}{l}
\sum_{i_{\alpha} j_{\alpha}}\left[\mu_{i_{\alpha} j_{\alpha}, \xi} \gamma_{i_{\alpha} j_{\alpha}, o c c .}^{m n}\right]^{2}+\sum_{a_{\beta} b_{\beta}}\left[\mu_{a_{\beta} b_{\beta}, \xi} \gamma_{a_{\beta} b_{\beta}, \text { unocc. }}^{m n}\right]^{2} \\
+2 \sum_{i_{\alpha} j_{\alpha}}\left[\mu_{i_{\alpha} j_{\alpha}, \xi} \gamma_{i_{\alpha} j_{\alpha}, \text { occ. }}^{m n}\right] \sum_{a_{\beta} b_{\beta}}\left[\mu_{a_{\beta} b_{\beta}, \xi} \gamma_{a_{\beta} b_{\beta}, \text { unocc. }}^{m n}\right]^{2}
\end{array}\right\}
$$


With respect to the same expression for the unrestricted case:

$$
\left\langle n\left|\mu_{\xi}\right| m\right\rangle\left\langle m\left|\mu_{\xi}\right| n\right\rangle=\frac{1}{4}\left\{\begin{array}{l}
\sum_{i_{\alpha} j_{\alpha}}\left[\mu_{i_{\alpha} j_{\alpha}, \xi} \gamma_{i_{\alpha} j_{\alpha}, o c c .}^{m n}\right]^{2}+\sum_{a_{\alpha} b_{\alpha}}\left[\mu_{a_{\alpha} b_{\alpha}, \xi} \gamma_{a_{\alpha} b_{\alpha}, \text { unocc. }}^{m n}\right]^{2} \\
+\sum_{i_{\beta} j_{\beta}}\left[\mu_{i_{\beta} j_{\beta}, \xi} \gamma_{i_{\beta} j_{\beta}, \text { occ. }}^{m n}+\sum_{a_{\beta} b_{\beta}}\left[\mu_{a_{\beta} b_{\beta}, \xi} \gamma_{a_{\beta} b_{\beta}, \text { unocc. }}^{m n}\right]^{2}\right. \\
+2 \sum_{i_{\alpha} j_{\alpha}}\left[\mu_{i_{\alpha} j_{\alpha}, \xi} \gamma_{i_{\alpha} j_{\alpha}, o c c .}^{m n}\right] \sum_{a_{\alpha} b_{\alpha}}\left[\mu_{a_{\alpha} b_{\alpha}, \xi} \gamma_{a_{\alpha} b_{\alpha}, \text { unocc. }}^{m n}\right] \\
+2 \sum_{i_{\alpha} j_{\alpha}}\left[\mu_{i_{\alpha} j_{\alpha}, \xi} \gamma_{i_{\alpha} j_{\alpha}, \text { occ. }}^{m n}\right] \sum_{i_{\beta} j_{\beta}}\left[\mu_{i_{\beta} j_{\beta}, \xi} \gamma_{i_{\beta} j_{\beta}, \text { occ. }}^{m n}\right] \\
+2 \sum_{i_{\alpha} j_{\alpha}}\left[\mu_{i_{\alpha} j_{\alpha}, \xi} \gamma_{i_{\alpha} j_{\alpha}, o c c .}^{m n}\right] \sum_{a_{\beta} b_{\beta}}\left[\mu_{a_{\beta} b_{\beta}, \xi} \gamma_{a_{\beta} b_{\beta}, \text { unocc. }}^{m n}\right] \\
+2 \sum_{a_{\alpha} b_{\alpha}}\left[\mu_{a_{\alpha} b_{\alpha}, \xi} \gamma_{a_{\alpha} b_{\alpha}, \text { unocc. }}^{m n}\right] \sum_{i_{\beta} j_{\beta}}\left[\mu_{i_{\beta} j_{\beta}, \xi} \gamma_{i_{\beta} j_{\beta}, \text { occ. }}^{m n}\right] \\
+2 \sum_{a_{\alpha} b_{\alpha}}\left[\mu_{a_{\alpha} b_{\alpha}, \xi} \gamma_{a_{\alpha} b_{\alpha}, \text { unocc. }}^{m n}\right] \sum_{a_{\beta} b_{\beta}}\left[\mu_{a_{\beta} b_{\beta}, \xi} \gamma_{a_{\beta} b_{\beta}, \text { unocc. }}^{m n}\right] \\
+2 \sum_{i_{\beta} j_{\beta}}\left[\mu_{i_{\beta} j_{\beta}, \xi} \gamma_{i_{\beta} j_{\beta}, \text { occ. }}^{m n}\right] \sum_{a_{\beta} b_{\beta}}\left[\mu_{a_{\beta} b_{\beta}, \xi} \gamma_{a_{\beta} b_{\beta}, \text { unocc. }}^{m n}\right]
\end{array}\right\},
$$

the terms implying the occupied-occupied $\beta$ and unoccupied-unoccupied $\alpha$ blocks are missing.

To recover missing terms containing $\beta$ indices in the closed-shell case, $\alpha$ and $\beta$ spinorbitals are considered equivalent. The closed-shell expression is multiplied by four because the state-to-state transition moment already includes a factor of two. (When written in terms of amplitudes, it is multiplied by $\sqrt{2}$ since amplitudes implicitly account for another $\sqrt{2}$ factor.)

For the spin-flip case, one needs to keep in mind that in both occupied $\alpha$ and unoccupied $\beta$ spaces two orbitals are identical. We could include a factor of two in the SF-state to SFstate transition moment recovering all terms of equation 6 as for the closed-shell case but it will double-count a lot of times 1-TDM elements belonging to the spin-flip active space. To dim out this effect, we chose to multiply equation 1 by $\sqrt{2}$ instead, recovering some of the terms but not all. This is an approximation that results shown in this publication seem to validate. Though, it is expected that oscillator strengths for excitations out of the spin-flip active space will be underestimated by a factor of two. Another possibility could be to exclude the spin-flip active space and only multiplying the other 1-TDM elements by a factor of two. This supposes an automatic identification of orbitals present in both spaces but this is clearly difficult to implement. Since the aim of any spin-flip methods is to provide a good description for all states belonging to the spin-flip active space while higher energy states are poorly described, we chose to use the simple $\sqrt{2}$ approximation in the simplified TD-DFT framework. Note that in Q-CHEM, the 1-TDM between SF states is 
constructed by copying occupied $\alpha$ and unoccupied $\beta$ blocks for both $\alpha$ and $\beta$ spins. This is equivalent as including a factor of two in transition moments. Thus, it should be expected that our oscillator strengths are half of ones computed by Q-CHEM when neglecting inherent differences between full and simplified schemes. 




FIG. S1: Singlet-triplet energy gaps for nine compounds obtained at both SF-TD-DFT/B3LYP level in comparison with the sTD-DFT method. $(\mathrm{MAD}=0.026 \mathrm{eV}$ and $\mathrm{RMSD}=0.099 \mathrm{eV})$

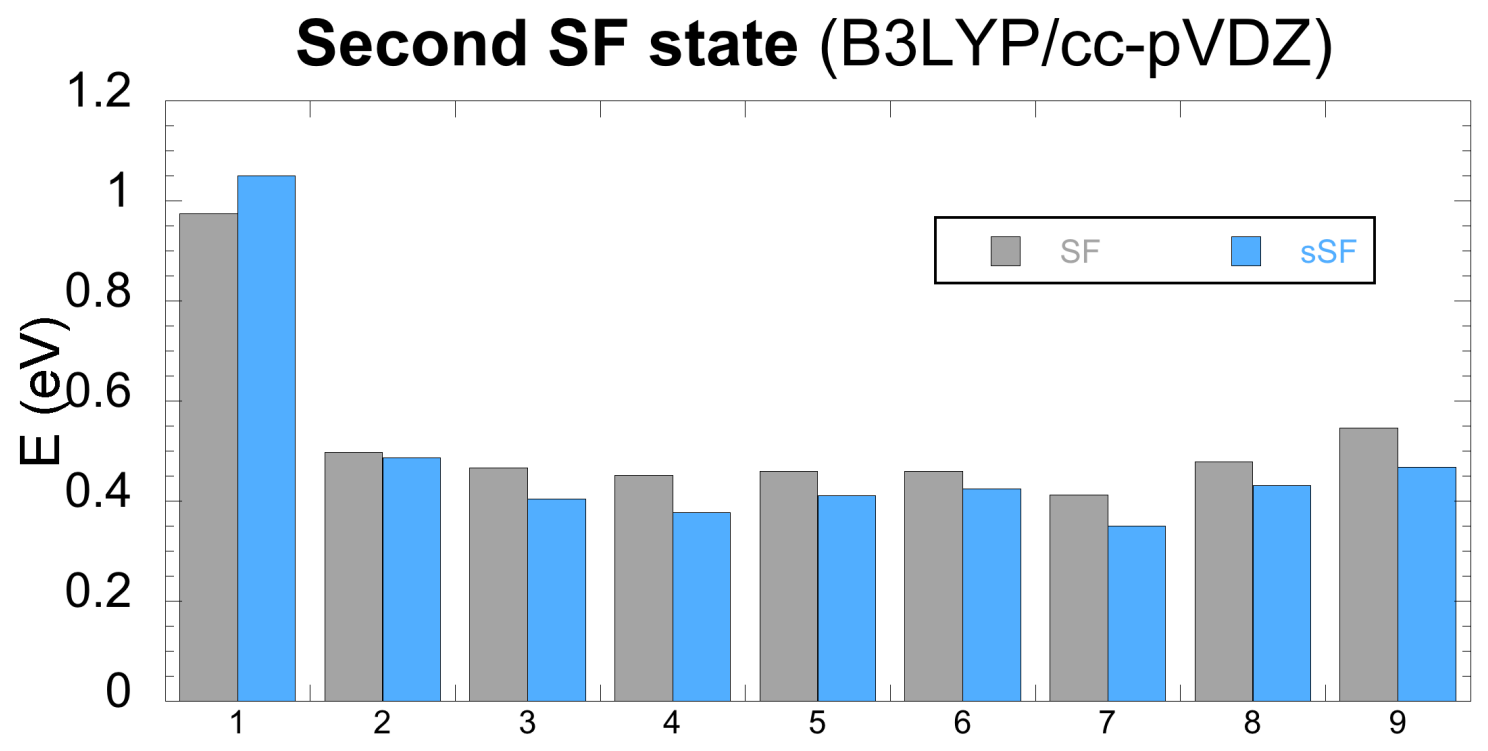

FIG. S2: Energy gaps between the first and second spin-flip states for nine compounds obtained at both SF-TD-DFT/B3LYP level in comparison with the sTD-DFT method. (MAD $=0.032 \mathrm{eV}$ and $\mathrm{RMSD}=0.059 \mathrm{eV})$ 


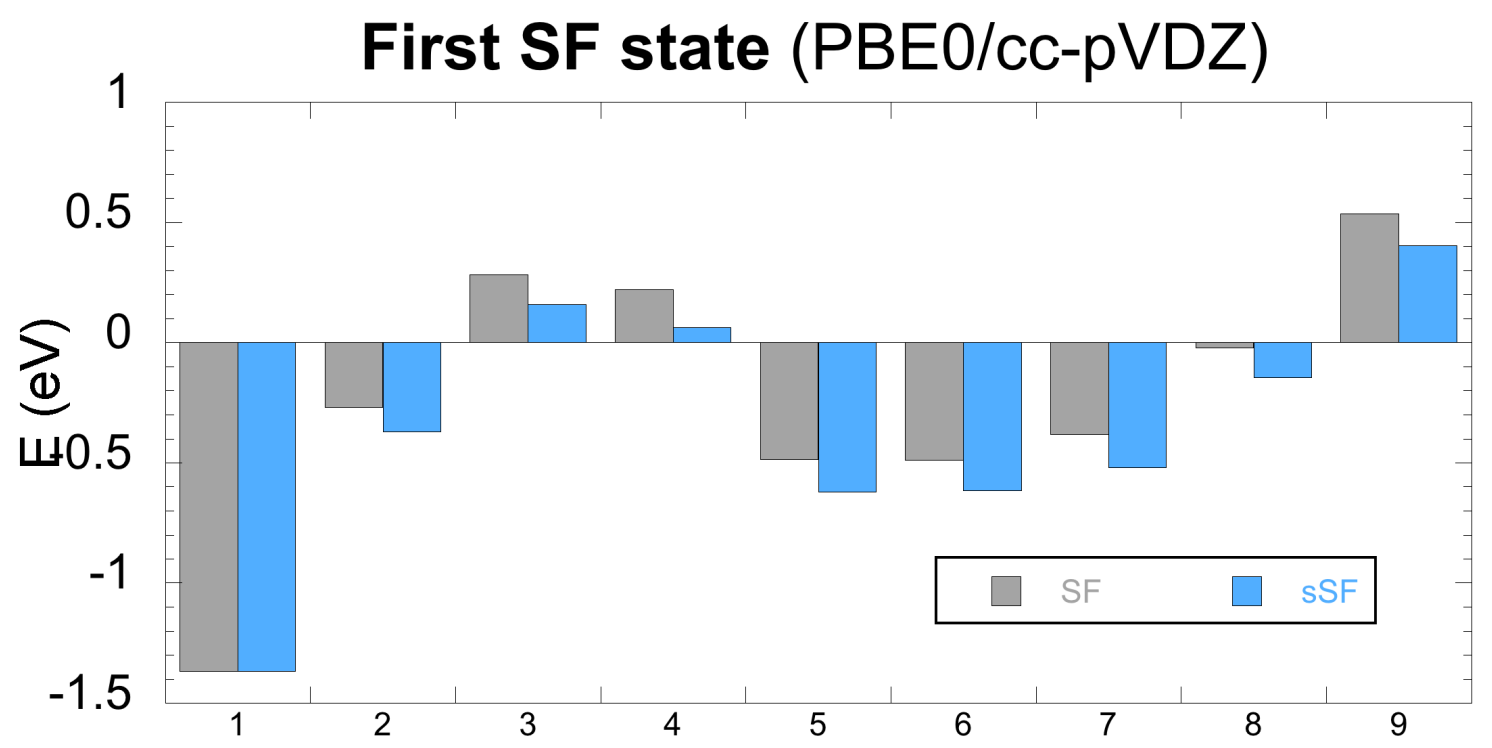

FIG. S3: Singlet-triplet energy gaps for nine compounds obtained at both SF-TD-DFT/PBE0 level in comparison with the sTD-DFT method. $(\mathrm{MAD}=0.029 \mathrm{eV}$ and $\mathrm{RMSD}=0.122 \mathrm{eV})$

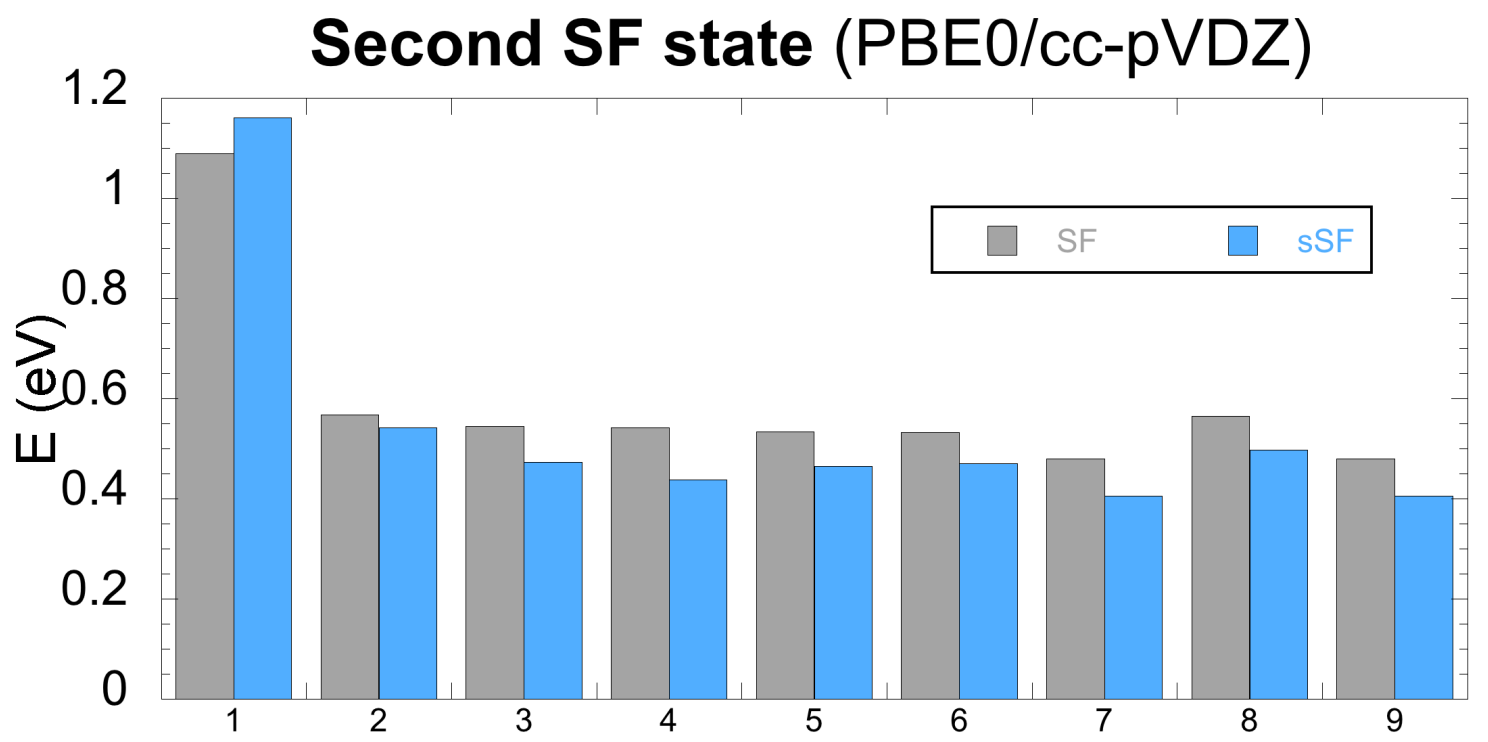

FIG. S4: Energy gaps between the first and second spin-flip states for nine compounds obtained at both SF-TD-DFT/PBE0 level in comparison with the sTD-DFT method. (MAD $=0.034 \mathrm{eV}$ and $\mathrm{RMSD}=0.072 \mathrm{eV})$ 


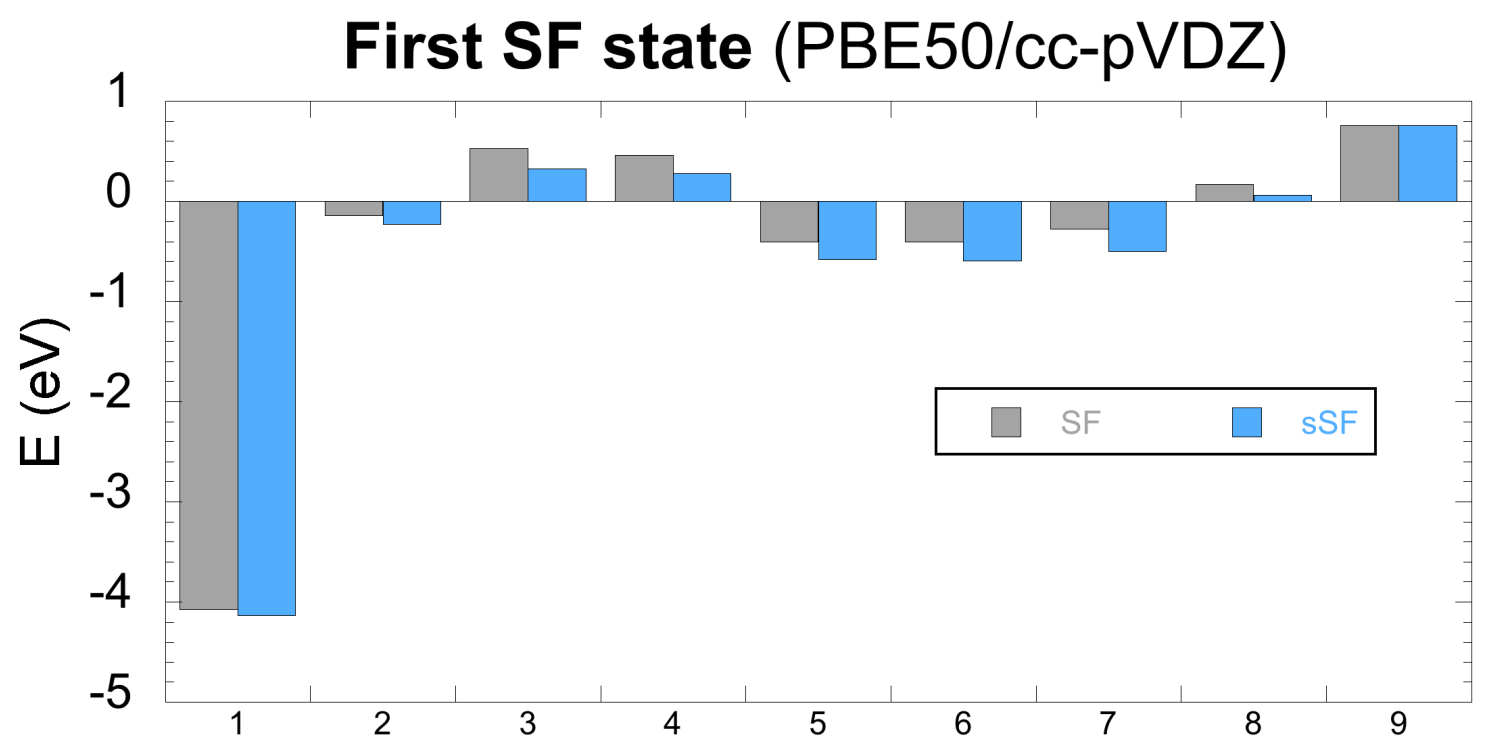

FIG. S5: Singlet-triplet energy gaps for nine compounds obtained at both SF-TD-DFT/PBE50 level in comparison with the sTD-DFT method. $(\mathrm{MAD}=0.064 \mathrm{eV}$ and $\mathrm{RMSD}=0.153 \mathrm{eV})$

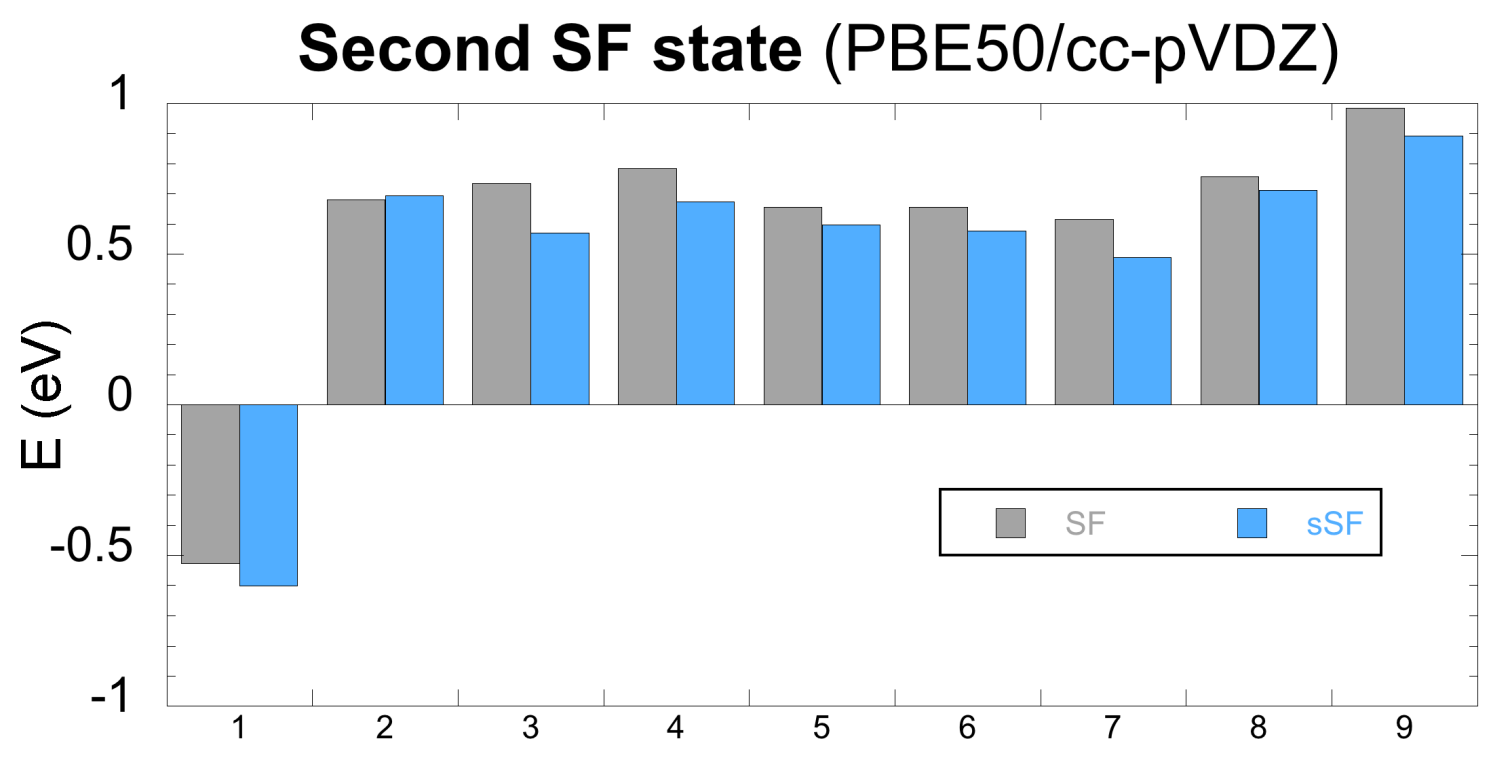

FIG. S6: Energy gaps between the first and second spin-flip states for nine compounds obtained at both SF-TD-DFT/PBE50 level in comparison with the sTD-DFT method. (MAD $=0.037 \mathrm{eV}$ and $\mathrm{RMSD}=0.095 \mathrm{eV}$ ) 


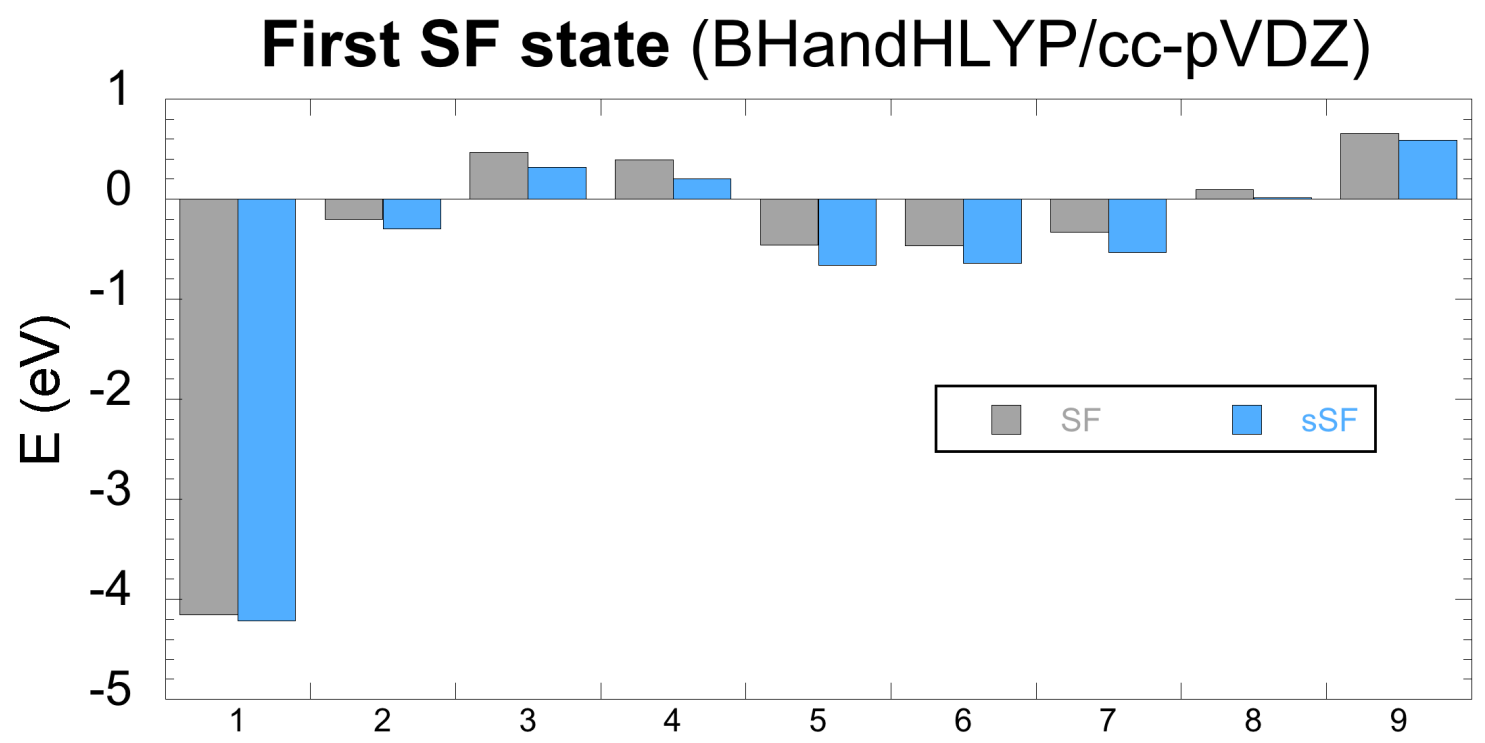

FIG. S7: Singlet-triplet energy gaps for nine compounds obtained at both SF-TDDFT/BHandHLYP level in comparison with the sTD-DFT method. (MAD $=0.053 \mathrm{eV}$ and RMSD $=0.145 \mathrm{eV})$

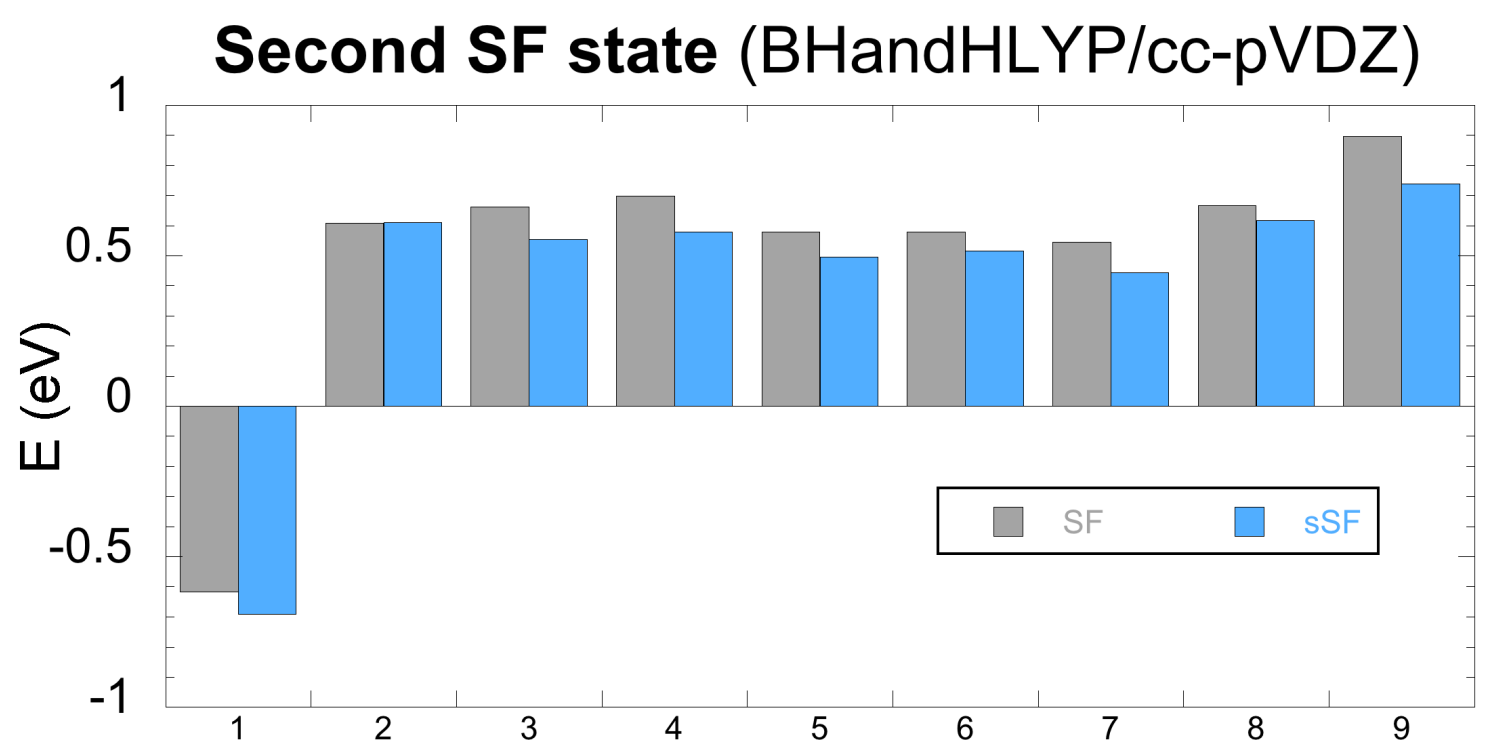

FIG. S8: Energy gaps between the first and second spin-flip states for nine compounds obtained at both SF-TD-DFT/BHandHLYP level in comparison with the sTD-DFT method. (MAD =0.034 $\mathrm{eV}$ and $\mathrm{RMSD}=0.094 \mathrm{eV}$ ) 